REVISTA PROYECCIONES $\mathrm{N}^{\circ} 10: 173-174$

Diciembre 1985 - ISSN 0716-0917

Jornada Matemáticas, Agosto 1985.

\title{
BASES CANÓNICAS DE HOMOLOGÍA
}

GUSTAVO LABBE MORALES*

Este trabajo está enmarcado en el área de la Geometría Analítica Compleja, y más especificamente, en el estudio de las Superficies de Riemann, esto es, variedades analíticas complejas de dimensión uno.

En el estudio de las superficies de Riemann, ha sido de gran interés poder hacer una clasificación de ellas. Así, por ejemplo, para el caso de superficies compactas y. conexas, se logró clasificarlas topológica mente, cuyas clases están dadas por el género. Esto es, una superficie de género g es una esfera con $g$ asas, módulo un homeomorfismo.

Para una clasificación conforme (o analitica), el problema se complica, pero se ha logrado establecer invariantes de la superficie, que

\footnotetext{
* Departamento de Ciencias Básicas, Universidad Arturo Prat, lquique.
} 
permiten clasificarlas conformemente. Por ejemplo, para una superficie de Riemann compacta y conexa definida por una ecuación algebraica, tenemos un invariante 11 amado matriz período. Esto es, suponemos que la superficie de Riemann $X$ de género $g$ es el dominio de definición de una función meromorfa n-valuada $W(z)$, definida por la ecuación $P(z, w)=0$, donde $P(z, w)$ es un polinomio irreducible con coeficiente complejos y variables $z, w$. A estas superficies $1 \mathrm{e}$ asociamos una matriz de orden $\mathrm{g} \times 2 \mathrm{~g}$, 11 amada matriz período, de la forma: $\pi=(A B)$, donde $A, B \in M(g \times g, \mathbb{C})$ con $\operatorname{det}(A) \neq 0$, que en la forma (IZ) se dice normalizada, con $Z=A^{-1} B$ simétrica con par te imaginaria definida positiva, 1lamada matriz de Riemann. La importancia de las matrices períodos la establece el teorema de Torel1i, en e1

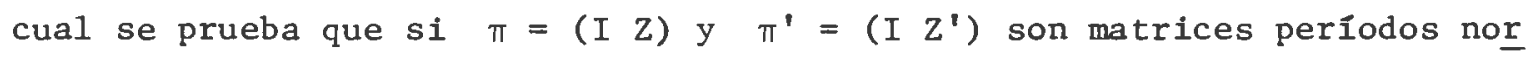
malizadas de superficies $X$ y $X^{\prime}$ respectivamente y si existe $N$ matriz simpléctica tal que $Z^{\prime}$ es obtenida normalizando $\pi N$, entonces $X X^{\prime}$ son con formemente equivalentes.

Los elementos de la matriz período se obtienen integrando una base de diferenciales abelianas de primera especie de X sobre una base ca nónica de homología de la superficie.

E1 objetivo principal de este trabajo, es mostrar un método que permite de manera combinatoria, obtener bases canónicas de homología de superficie. Para tal efecto, primero se mostrará el método tradicional para obtener bases de homología vía una descomposición celular de la superficie, para luego hacer un paralelo con el método combinatorio.

Este desarrollo se hará remitiéndose a dos ejemplos: 1a curva de Klein-Hurwit] $\quad \mathrm{W}^{7}=\mathrm{Z}(1-\mathrm{Z})^{2}$ y la curva de Fermat $\mathrm{W}^{\mathrm{q}}+\mathrm{Z}^{\mathrm{q}}=1$ (caso particular: $q=4$ ). 